Preprint of http://dx.doi.org/10.1063/1.3686290

\title{
DETERMINATION OF THE VELOCITY-CURVATURE RELATIONSHIP FOR UNKNOWN FRONT SHAPES
}

\author{
Scott I. Jackson and Mark Short \\ Shock and Detonation Physics Group, WX-9, LANL, Los Alamos, NM 87545
}

\begin{abstract}
Detonation Shock Dynamics (DSD) is a detonation propagation methodology that replaces the detonation shock and reaction zone with a surface that evolves according to a specified normal-velocity evolution law. DSD is able to model detonation propagation when supplied with two components: the normaldetonation-velocity variation versus detonation surface curvature and the surface edge angle at the explosiveconfiner interface. The velocity-curvature relationship is typically derived from experimental rate-stick data. Experimental front shapes can be fit to an analytic equation with an appropriate characteristic shape to examine detonation velocity-curvature variation computed from that analytic expression. However, in some complex explosive-confiner configurations, an appropriate functional form for the detonation front shape may be difficult to construct. To address such situations, we numerically compute the velocity-curvature variation directly from discrete experimental front-shape data using local rather than global fitting forms. The results are then compared to the global method for determining the velocity-curvature variation. The possibilities and limitations of such an approach are discussed.
\end{abstract}

Keywords: DSD, detonation shock dynamics, curvature, detonation

PACS: 47.40.Rs, 47.40.Nm, 82.40.Fp

\section{INTRODUCTION}

Measurement of the relationship between a detonation's normal velocity and its curvature is a key basis for Detonation Shock Dynamics theory (DSD), a surface propagation concept that replaces the detonation shock and reaction zone with a surface that evolves according to a specified normal-velocity evolution law [1]. The velocity-curvature $\left(D_{n}-\kappa\right)$ relationship is found by fitting assumed $\kappa\left(D_{n}\right)$ forms to digitized experimental front shape data, usually obtained from rate-stick tests.

Steady rate-stick configurations tend to exhibit gradual variations of $D_{n}$ and $\kappa$ near the charge center and rapid variations near the charge periphery, where edge effects are significant. This can be analyzed by fitting the front shape to the functional form,

$$
z(r)=-\sum_{i=1}^{n} A_{i} \ln \left[\cos \left[\eta \frac{\pi}{2} \frac{r}{R}\right]\right]^{i}
$$

where $z$ is the front height, $r$ is the local radius, and $R$ is the charge radius $[1,2]$. Equation 1 is fit globally across an entire experimental rate-stick front shape by varying parameters $A_{i}$ and $\eta$ where $0<\eta<1$. Parameter $n$ is typically chosen as 1 or 3 .

Once a global analytic fit $z(r)$ has been obtained, the normal velocity $D_{n}$ can then be found from

$$
D_{n}=\frac{D}{\sqrt{1+z^{\prime}}}
$$

where $z^{\prime}=d z / d r$ and $D$ is the axial detonation phase velocity. For a cylindrical rate stick, $\kappa$ can be expressed as

$$
\kappa=\frac{z^{\prime \prime}}{\left[1+\left(z^{\prime}\right)^{2}\right]^{3 / 2}}+\frac{z^{\prime}}{r \sqrt{1+\left(z^{\prime}\right)^{2}}}
$$

with $z^{\prime \prime}=d^{2} z / d r^{2}$. For a one-dimensional slab geometry, the second term of Eq. 3 would be zero. 
For complex explosive-confiner configurations, an appropriate functional form $z(r)$ to analyze detonation front shape may be difficult to construct. In this study we address such situations by locally computing the $D_{n}-\kappa$ relationship directly from discrete experimental front-shape data with no global assumptions of wave shape. This is done by locally fitting a parabolic form to local portions of each front shape.

The effectiveness of this technique is then explored by applying it to three experimental configurations: (1) a TATB-based explosive cylindrical rate stick for verification purposes, (2) a highly heterogenous ANFO-based cylindrical rate stick to resolve local and complex variations in wave shape, and (3) a TATB-based explosive slab whose wave shape cannot be fit by Eq. 1 .

\section{METHOD}

Experimental front record profiles consist of a list $Z$ composed of $n$ data points representing the detonation shape as it emerges from an explosive charge. Each point $\left(x_{i}, z_{i}\right)$ has an $x$ and $z$ coordinate, corresponding to its radial and vertical measurement location as shown in Fig. 1. All $x_{i}$ points lie inside the charge radius such that $-R \leq x_{i} \leq R$.

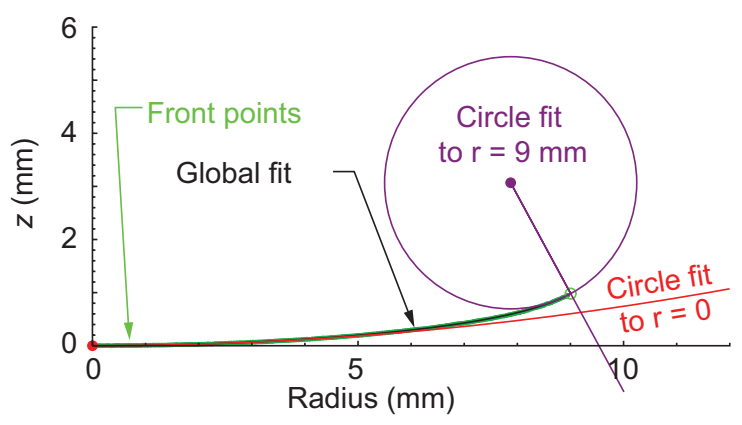

FIGURE 1. Front shape from a PBX 9502 rate stick.

The local fitting process starts by extracting a subset $S_{i}$ of points from $Z$ that lie within $\frac{\Delta r}{2}$ of a given front radius position $x_{c}$. A parabolic analytic form

$$
z_{p}(r)=a\left(r-X_{p}\right)^{2}+b\left(r-X_{p}\right)+Y_{p}
$$

is then fit to the front segment $S_{i}$ by varying fit parameters $a, b, X_{p}$, and $Y_{p}$. Using this local fit, along with Eqs. 2 and 3, then allows $D_{n}$ and $\kappa$ values to be calculated for the point $x_{c}$. This process is iterated for each point in $Z$, with $x_{c}$ taking the place of each value of $x_{i}$ along the front shape.

The parameter $\Delta r$ is an adjustable variable. Larger values fit across a greater portion of $Z$ and yield a higher quality fit, but do not detect rapid local variations. Smaller values recover these features, but are more sensitive to experimental and digitization errors due to a decreased number of fit points in $S_{i}$.

Near the edge of each front profile, it is not possible to center $S_{i}$ on $x_{c}$ as no experimental front shape data exists past the charge periphery. This results in decreased fit quality near $r=R$; for this reason we do not fit when $x_{c}$ is not within the center of $S_{i}$. Thus, an annular region of $Z$ where $R-\frac{\Delta r}{2} \leq x_{i} \leq R$ is not fit with this process.

\section{PBX 9502 RATE STICK VERIFICATION}

PBX 9502 is a plastic bonded explosive composed of $95 \%$ TATB and 5\% Kel-F binder. The explosive's morphology is very fine grained and yields very smooth front shape measurements. Data from an 18-mm-diameter unconfined PBX 9502 rate stick from Ref. [3] is shown in Fig. 1. Digitized front shape points are green and the corresponding global fit is black. Red and purple circles highlight the large variation in local wavefront curvature at the charge center and outer radius, respectively.

Figures 2 and 3 illustrate the variations in $D_{n}$ and $\kappa$ along the front as determined by the global fit method (black line) and the local fit methodology (data circles). Different color data points correspond to different values of $\Delta r$, as listed in the Fig. 3 legend. The local fit data agree with the global fit over most

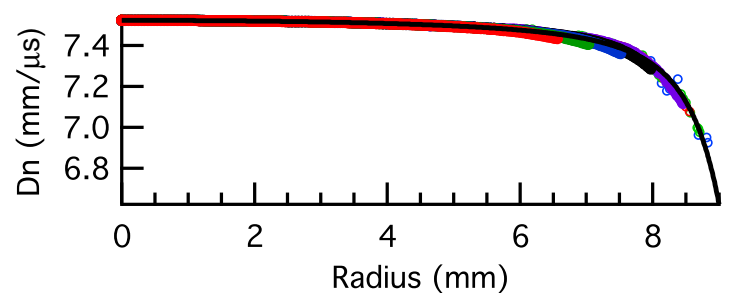

FIGURE 2. $\quad D_{n}$ versus $r$ for the front from Fig. 1 data.

the front. The $D_{n}$ fit quality is excellent for all values of $\Delta r$. The $\kappa$ fit experiences increased variation with decreasing $\Delta r$ due to the increased sensitivity of the second derivative to experimental noise. Near the charge edge however, lower values of $\Delta r$ are seen to 


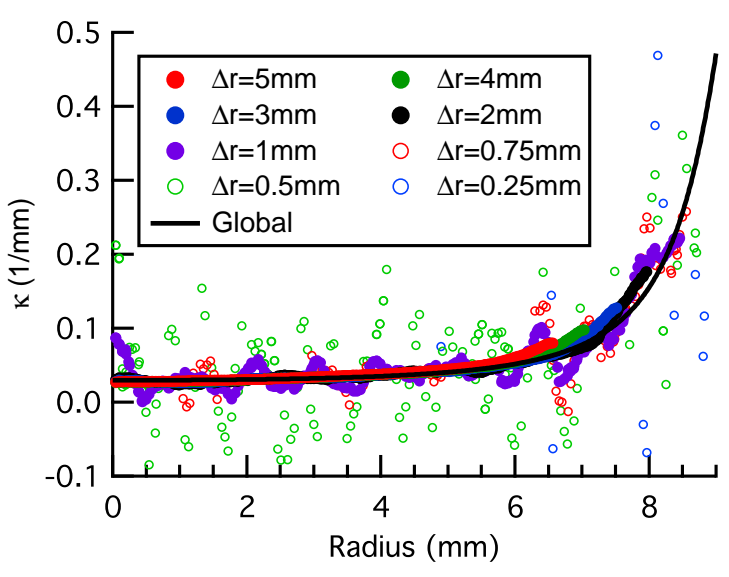

FIGURE 3. $\kappa$ versus $r$ for Fig. 1 data.

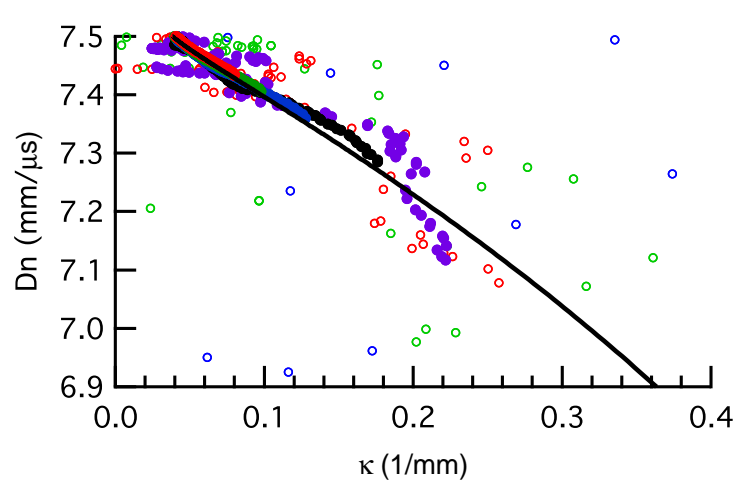

FIGURE 4. $D_{n}-\kappa$ data for Fig. 1 data.

more accurately match the global fit down to a breakdown limit, in this case $\Delta r=0.5 \mathrm{~mm}$, after which the local-fit $\kappa$ dissolves into scatter. Approximately 380 points (42 points/mm) were available for this front shape, yielding approximately 21 points in the local fit at breakdown. This highlights the importance of obtaining high-resolution front shape data.

\section{ANFO RATE STICK TEST}

Data from a cylindrical rate stick composed of ANFO confined by aluminum (test 12-305 from Ref. [4]) is shown in Figs. 5 and 6. The front breakout reflects the heterogenous nature of explosive, which is composed of approximately 2-mm-diameter ammonium-nitrate prills soaked with fuel oil. As in

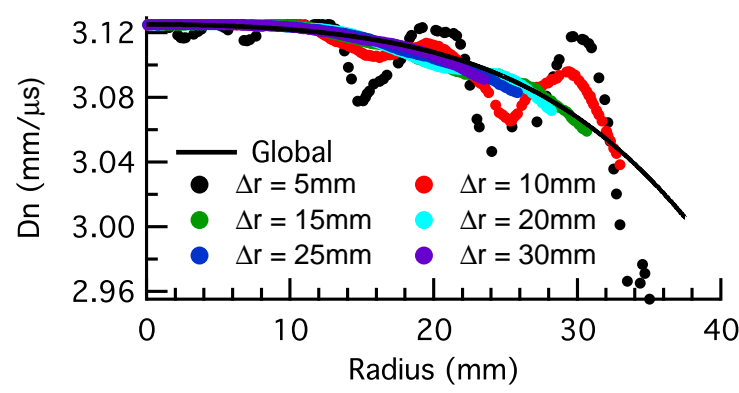

FIGURE 5. ANFO (a) front shape, (b) $D_{n}-r$, and (c) $\kappa-r$ data.

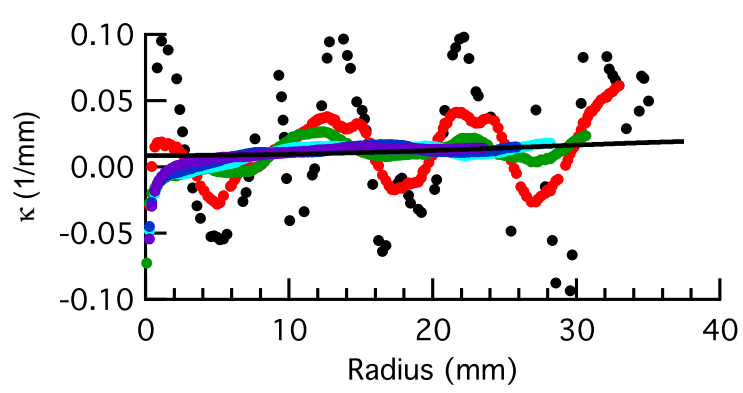

FIGURE 6. ANFO (a) front shape, (b) $D_{n}-r$, and (c) $\kappa-r$ data.

the previous example, larger values of $\Delta r(\geq 15 \mathrm{~mm})$ approximate the global fit well, while decreased values of $\Delta r(5$ and $10 \mathrm{~mm})$ recover the prill morphology.

\section{PBX 9502 SLAB TEST}

The rectangular slab test or "sandwich-style test," consists of an unconfined rectangular slab of explosive. The charge aspect ratio and boosting method are intended to isolate long-axis edge effects from the charge center during the test time [5].

An image of an unconfined slab test and detonation front shape is shown in Fig. 7 for a $151 \times 127 \times$ $8 \mathrm{~mm}$ thick slab of PBX 9502. Normally, detonation breakout is observed across the 8-mm-thick dimension [5]. This test was intended to verify the onedimensional nature of the geometry and measured the front shape across the 151-mm-long dimension as indicated by the black line denoting the streak measurement location. As the front shape is still evolv- 


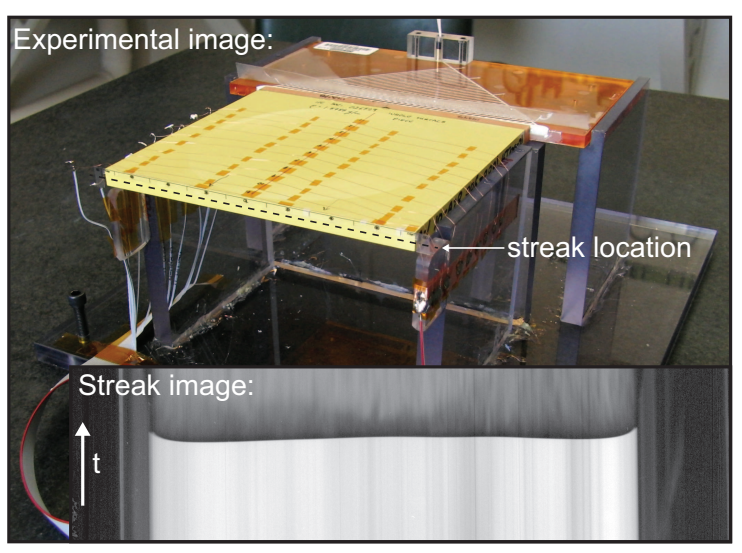

FIGURE 7. Slab (a) experiment and (b) front shape.

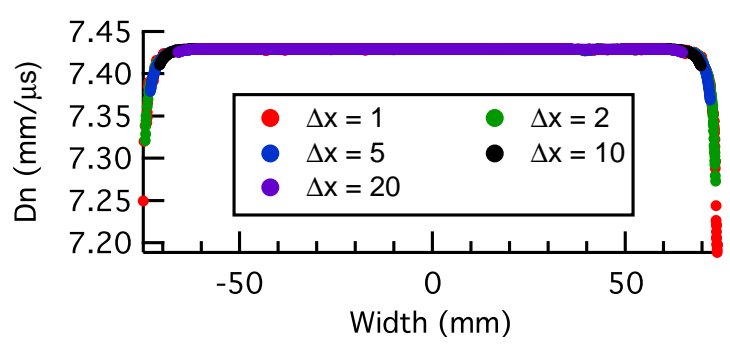

FIGURE 8. PBX $9502 \mathrm{slab}$ (a) $D_{n}-r$, and (c) $\kappa-r$ data.

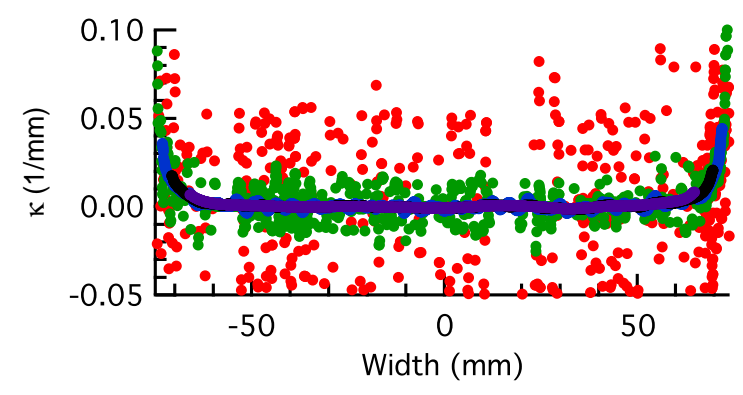

FIGURE 9. PBX $9502 \mathrm{slab}$ (a) $D_{n}-r$, and (c) $\kappa-r$ data.

ing over much of this axis, such front data is unsteady in nature, not well-represented by Eq. 1, and requires analysis by other means, such as the local fit method.

$D_{n}$ and $\kappa$ data from the local-fit method are shown in Fig. 9. The data indicate that minimal curvature is indeed present near the charge center and show that long-axis edge effects do not affect the flow in this region.

\section{CONCLUSIONS}

A numerical method was presented to measure local variations in the normal detonation velocity $D_{n}$ and surface curvature $\kappa$ from detonation front-shape data. Specifically, the method operates by locally fitting a parabolic equation to small sections of a front. Such an approach allows variation of detonation velocity and curvature to be examined for explosive configurations with no assumptions of global wave shape.

The methodology was verified and compared to a prior technique for $D_{n}-\kappa$ variation determination that involves globally fitting an analytic form across the entire front shape. Front shape data from a heterogeneous ANFO rate stick and a non-standard slabgeometry test were also analyzed. In general, data from the local-fit method agreed with the global fit method. Reduction of the fitting section size was found to increase the method's sensitivity to local wave shape variations and also to experimental noise.

\section{ACKNOWLEDGEMENTS}

This effort was funded by the Department of Energy. Support was provided through programs administered by Dan Hooks, Jim Koster, Rick Martineau, and David Robbins.

\section{REFERENCES}

1. Bdzil, J., J. Fluid Mech., 108, 195-226 (1981).

2. Catanach, R., and Hill, L., "Diameter Effect Curve and Detonation Front Curvature Measurements for ANFO," in Shock Compression of Condensed Matter, American Institute of Physics, 2001, pp. 906-909.

3. Hill, L., Bdzil, J., Davis, W., and Critchfield, R., "PBX 9502 Front Curvature Rate Stick Data: Repeatability and the Effects of Temperature and Material Variation," in Ninth Symposium (Int.) on Detonation, Office of Naval Research, 2006, pp. 331-341.

4. Jackson, S., Kiyanda, C., and Short, M., "Experimental Observations of Detonation in Ammonium-NitrateFuel-Oil (ANFO) Surrounded by a High-Sound-Speed, Shockless, Aluminum Confiner," in Proceedings of the 33rd International Combustion Symposium, The Combustion Institute, 2010, pp. 2219-2226.

5. Hill, L., and Aslam, T., "The LANL DetonationConfinement Test: Prototype Development and Sample Results," in Shock Compression of Condensed Matter, American Institute of Physics, 2003, pp. 847-850. 